\title{
ON THE COMPUTATION OF THE CLASS NUMBERS OF SOME CUBIC FIELDS
}

\section{MANNY SCAROWSKY and ABRAHAM BOYARSKY}

\author{
Department of Mathematics \\ Loyola Campus \\ Concordia University \\ Montreal, Canada H4B 1R6 \\ (Received December 10, 1985)
}

ABSTRACT. Class numbers are calculated for cubic fields of the form $x^{3}+12 A x-12=0$, $A>0$, for $1 \leq \mathrm{a} \leq 17$, and for some other values of $A$. These fields have a known unit, which under certain conditions is the fundamental unit, and are important in studying the Diophantine Equation $x^{3}+y^{3}+z^{3}=3$.

KEY WORDS AND PHRASES. Class numbers, cubic fields, Diophantine equation. 1980 AMS SUBJECT CLASSIFICATION CODES. 12A04, $12 A 50$.

1. INTRODUCTION AND SOME THEOREMS.

We consider the cubic fields defined by an equation of the form

$$
f(x)=x^{3}+12 A x-12=0,
$$

where $A>0$. The field defined by this equation is important because it is related to the Diophantine equation $x^{3}+y^{3}+z^{3}=3$ when $A=9 a^{2}[1]$. Equation (1.1) is clearly irreducible, and as $f(x)$ is increasing, it defines a real cubic field $\mathrm{K}$ (with two complex conjugates) with exactly one fundamental unit. Let $\theta$ be the real root of $(1.1)$. We write $K=Q(\theta)$. Note that $0<\theta<1$. Also $n=\frac{\theta^{3}}{12}=1-A \theta$ defines a unit of $K$. As $0<\eta<1$, we have $\theta<\frac{1}{A}$. The discriminant of $f(x)$ is $D=-2^{4} \cdot 3^{3}\left(16 A^{3}+9\right)$. As $f(x)$ is an Eisenstein polynomial with respect to 3 , we have (3) $=q^{3}$. Also as $\frac{6}{\theta}$ satisfies $x^{3}-36 \mathrm{Ax}-18=0$, we see that for the same reason (2) $=p^{3}$, and as $\frac{6}{\theta}=6 A+\frac{\theta^{2}}{2}$ we see that $\frac{\theta^{2}}{2} \varepsilon 0_{K}$, the ring of integers of $K$. Thus the descriminant, $D$, of $K$, divides $-2^{2} \cdot 3^{3}\left(16 A^{3}+9\right)$. We now state: THEOREM 1. In $K$, the discriminant $D=\frac{-2^{2} \cdot 3^{3}\left(16 A^{3}+9\right)}{q^{2}}$ where $q^{2}$ is the largest square, prime to 3 , dividing $D$. The unit $n$ is never a cube, and if $q=1$ or $q=5$ then $n$ is the fundamental unit except when $A=1$. The class-number $h$, of $K$, is divisible by 3 . The primes $p_{i}$ dividing $D$ (except for 2 and 3 ) ramify as 
$\left(p_{i}\right)=p_{i}^{2} q_{i}$. A basis for $o_{K}$ is given by $\theta_{0}=1, \theta_{1}=\frac{\theta^{2}}{2}, \theta_{2}=\frac{16 A^{2}+3 \theta+2 A \theta^{2}}{3^{i} q}$, $\left(B^{i}=(3, A)\right)$.

As the proof is similar to the proof of the corresponding theorem in [1], we omit it, as well as the proof of the following two theorems, also in [1].

THEOREM 2. If the 3-component of the class-group of $k$ is a direct product of cyclic groups of order 3 , then

$$
x^{3}+12 A x-12=4 z^{3}
$$

has no solutions.

Corollary: If $3 \mathrm{Ih}$, then (1.2) has no solutions.

THEOREM 3. If $(h, 2)=1$, and $q=1$, then solving $x^{3}+12 A x-12=y^{2}$ is equivalent to solving $-\mathrm{AG}^{4}-2 \mathrm{G}^{3} \mathrm{H}+3 \mathrm{H}^{4}=-1$ (This has no solutions (mod $\mathrm{p}$ ) for small primes $\mathrm{p}$, e.g. $A=14, p=5$ ).

2. NUMERICAL COMPUTATIONS.

$$
\text { We note that } \lim _{s \rightarrow 1+} \frac{\zeta_{k}(s)}{\zeta(s)}=\frac{4 \pi \log \varepsilon \cdot h}{2 \sqrt{2^{2} \cdot 3^{3}\left(16 A^{3}+9\right) / q^{2}}}
$$

where $\varepsilon>1$ is the fundamental unit of $K$. As in [2], the left-hand side of (2.1) can be expressed as $f=\lim _{\mathrm{P} \rightarrow \infty} f_{p}=\lim _{\mathrm{P} \rightarrow \infty} \prod_{\mathrm{I}}^{\mathrm{P}} \mathrm{f} f(\mathrm{p})$ where

$$
f(p)= \begin{cases}\frac{p}{p-1} & \text { if } p \text { ramifies }\left(\left(p_{1}\right)=p_{i}^{2} q_{i}\right) \\ \frac{p^{2}}{p^{2}+p+1} & \text { if } p \text { remains inert } \\ \frac{p^{2}}{p^{2}-1} & \text { if }(p)=p q \\ \left(\frac{p}{p-1}\right)^{2} & \text { if } p \text { splits completely }\end{cases}
$$

Hence (2.1) implies that approximately,

for $P$ sufficiently large.

$$
h=\frac{\sqrt{27\left(16 A^{3}+9\right)}}{\pi \cdot q \cdot \log \varepsilon} \mathrm{f}_{P}
$$

\begin{tabular}{|c|c|c|c|c|}
\hline$\underline{A}$ & $-D / 2^{2} \cdot 3^{3}$ & $\underline{\mathbf{h}}$ & $\underline{A}$ & $-D / 2^{2} \cdot 3^{3}$ \\
\hline 1 & $5^{2}$ & 6 & 19 & $7 \cdot 15679$ \\
\hline 2 & 137 & 3 & 20 & $7 \cdot 18287$ \\
\hline 3 & $3^{2} \cdot 7^{2}$ & 6 & 21 & $3^{2} \cdot 5 \cdot 37 \cdot 89$ \\
\hline 4 & 1033 & 6 & 22 & $347 \cdot 491$ \\
\hline 5 & $7^{2} \cdot 41$ & 3 & 23 & 194681 \\
\hline 6 & $3^{2} \cdot 5 \cdot 7 \cdot 11$ & 21 & 24 & $3^{2} \cdot 7 \cdot 3511$ \\
\hline
\end{tabular}

For Table 1, the product in (2.2) was calculated for $P=P(2027)$, (at intervals of 50), where $P(i)$ indicates the $i^{\text {th }}$ prime, and $1 \leq A \leq 36$ :

\section{TABLE 1}




$\begin{array}{llllll}\mathrm{A} & \frac{-\mathrm{D} / 2^{2} \cdot 3^{3}}{23 \cdot 239} & \underline{\mathrm{h}} & \underline{\mathrm{A}} & \frac{-\mathrm{D} / 2^{2} \cdot 3^{3}}{29 \cdot 37 \cdot 233} & \underline{\mathrm{h}} \\ 7 & 23 & 25 & 22 \\ 8 & 59 \cdot 139 & 18 & 26 & 5^{2} \cdot 7 \cdot 1607 & 15 \\ 9 & 3^{2} \cdot 1297 & 12 & 27 & 3^{2} \cdot 7 \cdot 4999 & 54 \\ 10 & 7 \cdot 2287 & 27 & 28 & 11 \cdot 37 \cdot 863 & 78 \\ 11 & 5 \cdot 4261 & 24 & 29 & 359 \cdot 1087 & 48 \\ 12 & 3^{2} \cdot 7 \cdot 439 & 24 & 30 & 3^{2} \cdot 23 \cdot 2087 & 72 \\ 13 & 7 \cdot 5023 & 48 & 31 & 5 \cdot 7 \cdot 13619 & 162 \\ 14 & 43913 & 21 & 32 & 17 \cdot 3084 & 78 \\ 15 & 3^{2} \cdot 17 \cdot 353 & 36 & 33 & 7 \cdot 82143 & 114 \\ 16 & 5 \cdot 13109 & 36 & 34 & 7 \cdot 89839 & 87 \\ 17 & 7 \cdot 11 \cdot 1021 & 48 & 35 & 686009 & 75 \\ 18 & 3^{2} \cdot 10369 & 36 & 36 & 3^{2} \cdot 5 \cdot 53 \cdot 313 & 156\end{array}$

In a11 the cases above except when $A=1$ or $A=5, \quad \eta=\frac{1}{\varepsilon}$ is the fundamental unit of $K$. When $A=1,5, n=\varepsilon^{-2}$. $K$ is a pure cubic field if and only if $A=1$ or $\mathrm{A}=3$.

Also because of the equivalence of (1.2) with the Diophantine equation $x^{3}+y^{3}+z^{3}=3$ when $A=9 a^{2}$, the class-numbers of $K$ were calculated using (2.2) for $1 \leq a \leq 17$ (Actually Cassels has shown that for solutions of (1.2) to exist in this case, one must have $3 / a$ [3]). While most of the values obtained in this way were approximate, perhaps congruence conditions may be used to find them exactly, or perhaps they may be of use in regards to Brauer-Siegel Theorem, so we list them in Table 2. (The Brauer-Siege1 Theorem applied here states $\log \mathrm{h} \sim \frac{3}{2} \log \mathrm{A}-\log \mathrm{q}$ ).

TABLE 2

$\begin{array}{llll}\text { a } & -\mathrm{D} / 2^{2} \cdot 3^{5} & I\left(P(I) \text { Is the } I^{\text {th }} \text { prime }\right) & \underline{\text { h }} \\ 1 & 1297 & 8303 & 12 \\ 2 & 5 \cdot 53 \cdot 313 & 14903 & 156 \\ 3 & 5 \cdot 188957 & 10803 & 216 \\ 4 & 5380417 & 4303 & 420 \\ 5 & 3557 \cdot 5693 & 2201 & 789 \text { (*) } \\ 6 & 37 \cdot 241.6781 & 3003 & 1410 \\ 7 & 5 \cdot 30494621 & 1002 & 3285 \\ 8 & 53 \cdot 17 \cdot 29 \cdot 37 \cdot 149 & 1002 & 873 \\ 9 & 17 \cdot 40514561 & 1002 & 3549 \\ 10 & 181 \cdot 1361 \cdot 5261 & 1002 & 6999 \\ 11 & 89 \cdot 25797113 & 212 & 6753 \\ 12 & 5 \cdot 23761 \cdot 32573 & 212 & -15999 \\ 13 & 5 \cdot 8821 \cdot 141833 & 212 & 21864\end{array}$




$\begin{array}{llll}\text { a } & \frac{-\mathrm{D} / 2^{2} \cdot 3^{5}}{14} & \underline{I\left(P(I) \text { Is the } I^{\text {th }} \text { prime }\right)} & \underline{h} \\ 14 & 37 \cdot 263737261 & 212 & 10062 \\ 15 & 1193 \cdot 2381 \cdot 5197 & 212 & 22653 \\ 16 & 35801 \cdot 607337 & 212 & 16764 \\ 17 & 5^{2} \cdot 1251291577 & 212 & 4644\end{array}$

The second column gives the factorization of $-D / 2^{2} \cdot 3^{5}$.

(*) For $a \geq 5$, the values of $h$ should be considered as estimates, but are probably accurate within $\frac{1}{2} \%$.

Al1 computations were done at Concordia University, Loyola Campus, on the Cyber 170, Mode1 835 .

ACKNOWLEDGEMNT. The research of Abraham Boyarsky was supported by an NSERC Grant \#A9072 and an FCAC grant from the Quebec Department of Education.

\section{REFERENCES}

1. SCAROWSKY, M., On units of Certain Cubic Fields and the Diophantine Equation $x^{3}+y^{3}+z^{3}=3$, Proc. Amer. Soc., 91(3), 1984, 351-356.

2. SHANKS, D., The Simplest Cubic Fields, Math. of Comp., 28(1974), 1137-1152.

3. CASSELS, J.W.S., Personal communication. 


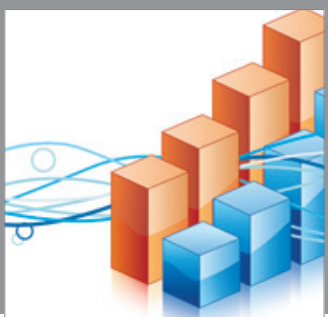

Advances in

Operations Research

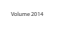

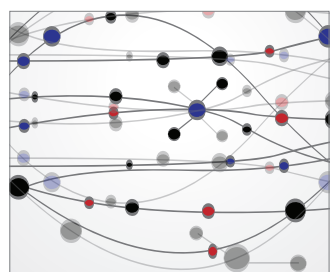

\section{The Scientific} World Journal
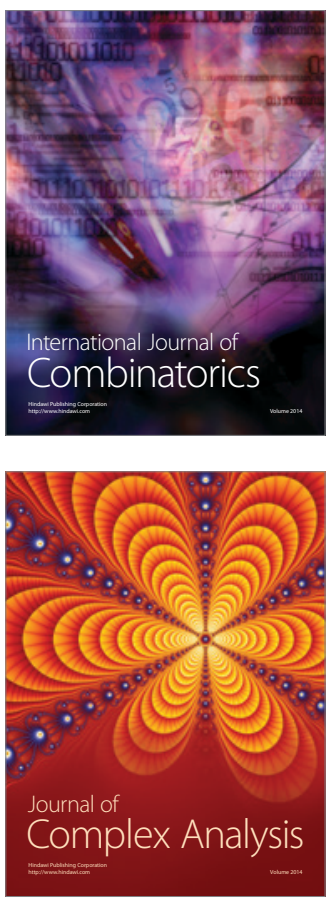

International Journal of

Mathematics and

Mathematical

Sciences
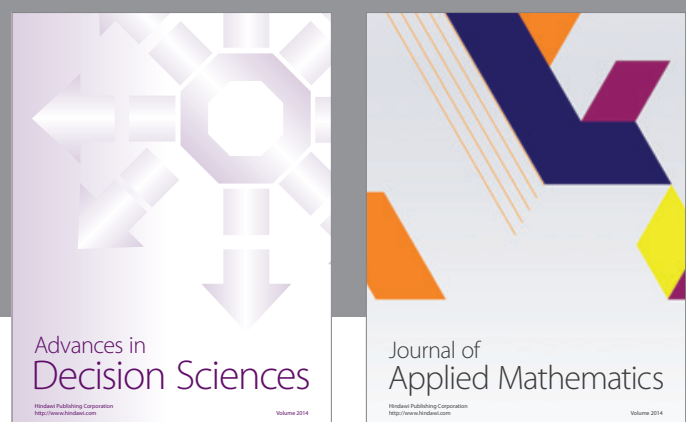

Journal of

Applied Mathematics
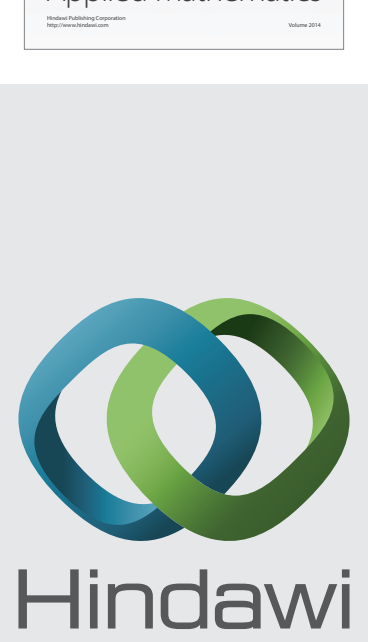

Submit your manuscripts at http://www.hindawi.com
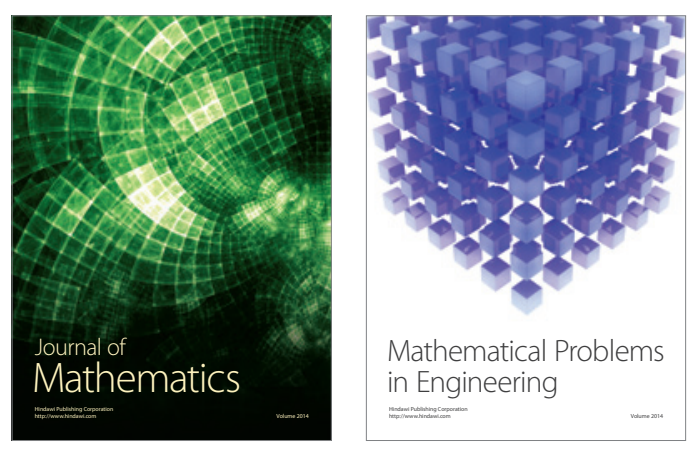

Mathematical Problems in Engineering
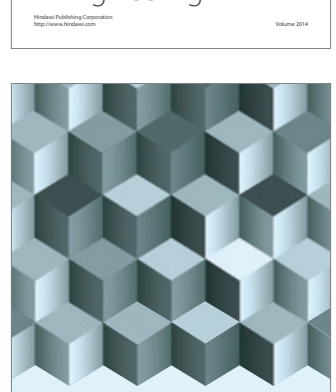

Journal of

Function Spaces
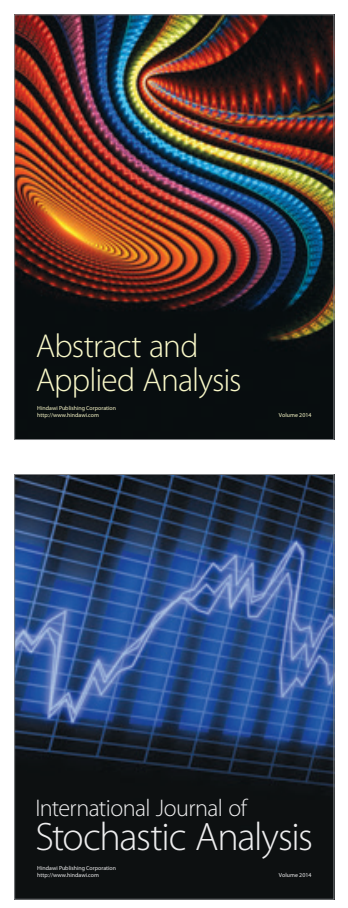

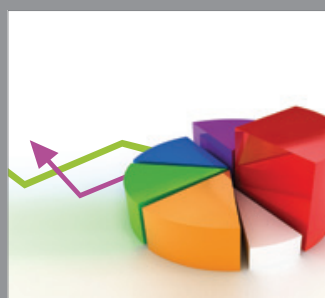

ournal of

Probability and Statistics

Promensencen
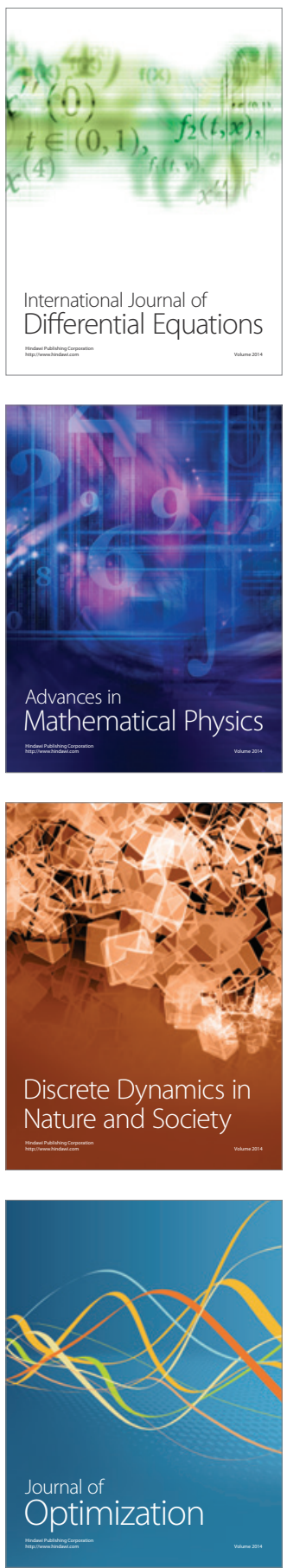